\title{
COVID-19 Pandemic: Power Law Spread and Flattening of the Curve
}

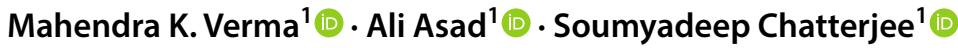

Received: 13 April 2020 / Accepted: 15 May 2020 / Published online: 31 May 2020

(C) Indian National Academy of Engineering 2020

\begin{abstract}
In this paper, we analyze the real-time infection data of COVID-19 epidemic for nine nations. Our analysis is up to May 04, 2020. South Korea, China, Italy, France, Spain, and Germany have either flattened or close to flattening their epidemic curves. USA and Japan have transitioned to a linear regime, while India is still in a power-law phase. We argue that the transition from an exponential regime to a succession of power-law regimes is a good indicator for flattening of the epidemic curve. We also argue that lockdowns, long-term community transmission, and the transmission by asymptomatic carriers traveling long distances may be inducing the power-law growth of the epidemic.
\end{abstract}

Keywords COVID-19 · Epidemic spread · Power law growth

\section{Introduction}

COVID-19 pandemic has caused major disruptions in the world. As of May 9, 2020, it has infected approximately 4 million humans, killed more than 0.27 million individuals, and has brought most of the world to a standstill in lockdowns (WorldOMeter 2020; Johns Hopkins University 2020). Hence, modeling and forecast of this epidemic is of critical importance. In this paper, we analyze the publicly available data set given in the WorldOMeter (2020). The data show that countries that have achieved flattening of the epidemic curve exhibit power law growth before saturation. This feature can be used as an important diagnostic for flattening of the epidemic curve.

Epidemiologists have made various models for understanding and forecasting epidemics. Kermack and McKendrick (1927) constructed one of the first models, called SIR model, for epidemic evolution. Here, the variables $S$ and $I$ describe respectively the numbers of susceptible and infected individuals. The variable $R$ represents the removed individuals who have either recovered or died. The SIR model has been generalized to SEIR model that includes exposed individuals, $E$, who are infected but not yet infectious (Bjørnstad 2018; Daley and Gani 2001).

Mahendra K. Verma

mkv@iitk.ac.in

1 Department of Physics, Indian Institute of Technology Kanpur, Kanpur 208016, India
SARS-CoV-2 is an extremely contagious virus. In addition, many infected individuals, called asymptomatic carriers, who show mild or no symptoms of infection have contributed significantly to the spread of the epidemic unwittingly ( $\mathrm{Li}$ et al. 2020). Hence, modelling COVD-19 requires more complex models of epidemiology, including features of quarantine, lockdowns, stochasticity, interactions among population pockets, etc. Note that quarantines and lockdowns help in suppressing the maximum number of infected individuals; such steps are critical for the epidemic management with limited public health resources. The saturation or flattening of the curve in China is attributed to strong lockdowns.

For COVID-19 epidemic, some of the new models have managed to provide good forecasts that appears to match with the data. Peng et al. (2020) constructed a seven-variable model (including quarantined and death variables) for epidemic spread in China and predicted that the daily count of exposed and infectious individuals will be negligible by March 30, 2020. Their predictions are in good agreement with the present data. Chinazzi et al. (2020) studied the effects of travel restrictions on the spread of COVID-19 in China and in the world, and Hellewell et al. (2020) performed feasibility studies of controlling COVID-19 epidemic by isolation. Mandal et al. (2020) constructed a India-specific model for devising intervention strategies; they focussed on four metros-Delhi, Mumbai, Kolkata, and Bengaluru—along with intercity connectivity. To account for spatio-temporal behavour, 
Min et al. (2013) simulated how a disease could spread within a network with different mixing styles, and showed that the average epidemic size and speed depend critically on network parameters. In addition, there are many epidemic models that are inspired by population growth models (Daley and Gani 2001; Wu et al. 2020). There are several other models designed to understand the spread of COVID-19 (Labadin et al. 2020; Shim et al. 2020; Shayak et al. 2020). In another interesting of analysis of COVID-19 epidemic, Schüttler et al. (2020) argued that $I(t)$ or total death count could be modelled using the error function.

We observed that along with us, several other researchers have also reported power-law growth after the exponential regime (Ziff and Ziff 2020; Komarova and Wodarz 2020; Manchein et al. 2020; Blasius 2020; Cherednik and Hill 2020). Hence, this conjecture is quite robust. We will detail our results in the next section.

\section{Data Analysis and Results}

In this paper, we report our results based on a comprehensive data analysis of nine major countries-China, USA, Italy, France, Spain, Germany, South Korea, Japan and India. We chose the above nations because of the large numbers of positive cases here. For our analysis, we employed the realtime data available at WorldOMeter (2020).

We digitized the data up to May 04, 2020 and studied the temporal evolution of the cumulative count of infected individuals, which is denoted by $I(t)$, where $t$ is time in days. In Fig. 1 we plot the time series of $I(t)$ and its derivative $\dot{I}(t)$ in semi-logy format using red and blue curves respectively. The derivatives $\dot{I}(t)$ have been computed using Python's gradient function. The starting dates for the plots are listed in Table 1.

No single function appears to fit with $I(t)$, hence we employ different functions to fit at different time intervals. As is well-known, in the early phase, the growth is exponential, but it transitions to power laws subsequently. Hence, we employ exponential function and polynomials for constructing best-fit curves to different parts of $I(t)$. The best-fit
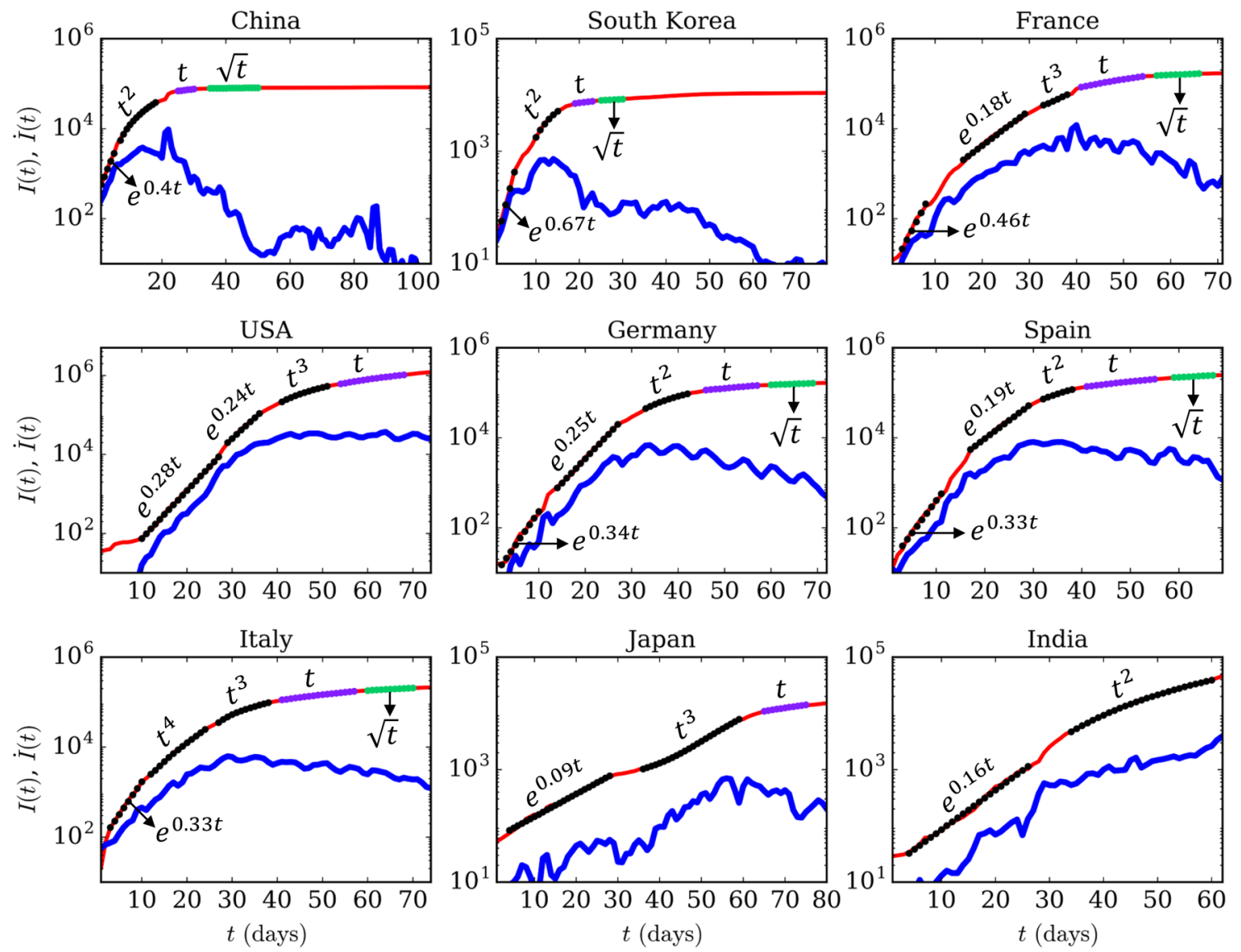

Fig. 1 (color online) For the COVID-19 epidemic, the semi-logy plots of total infected individuals $(I(t))$ vs. time $(t)$ (red thin curves) for the nine nations. We also plot $\dot{I}(t)$ vs. $t$ (blue thick curves). The black dotted curves represent the best-fit curves. For the best-fit functions, refer to Table 1 
Table 1 For the COVID-19 data for various countries, the best-fit functions and the respective relative errors for various stages of evolution shown in Fig. 1. The figure also exhibits the respective best-fit curves

\begin{tabular}{|c|c|}
\hline Countries (Start date) & Best-fit functions and errors \\
\hline \multirow[t]{4}{*}{ China (January 22) } & 1) $380 \mathrm{e}^{0.40 t}( \pm 2.2 \%)$ \\
\hline & 2) $89 t^{2}+750 t-4100( \pm 2.8 \%)$ \\
\hline & 3) $1400 t-34,000( \pm 0.64 \%)$ \\
\hline & 4) $2200 \sqrt{t}+65,000( \pm 0.36 \%)$ \\
\hline \multirow[t]{4}{*}{ South Korea (February 18) } & 1) $15 \mathrm{e}^{0.67 t}( \pm 2.2 \%)$ \\
\hline & 2) $9.7 t^{2}+430 t-3500( \pm 1.5 \%)$ \\
\hline & 3) $160 t+4000( \pm 0.67 \%)$ \\
\hline & 4) $880 \sqrt{t}+3600( \pm 0.085 \%)$ \\
\hline \multirow[t]{5}{*}{ France (February 24) } & 1) $5.4 \mathrm{e}^{0.46 t}( \pm 11 \%)$ \\
\hline & 2) $110 \mathrm{e}^{0.18 t}( \pm 6.5 \%)$ \\
\hline & 3) $6.9 t^{3}-360 t^{2}+4200 t+40,000( \pm 1.6 \%)$ \\
\hline & 4) $4600 t-11 \times 10^{4}( \pm 1.1 \%)$ \\
\hline & 5) $27000 \sqrt{t}-51,000( \pm 0.45 \%)$ \\
\hline \multirow[t]{4}{*}{ USA (February 21) } & 1) $4.6 \mathrm{e}^{0.28 t}( \pm 4.1 \%)$ \\
\hline & 2) $17 \mathrm{e}^{0.24 t}( \pm 3.5 \%)$ \\
\hline & 3) $8.5 t^{3}-970 t^{2}+67,000 t-15 \times 10^{5} \quad( \pm 0.46 \%)$ \\
\hline & 4) $30,000 t-1 \times 10^{6}( \pm 0.44 \%)$ \\
\hline \multirow[t]{5}{*}{ Germany (February 23) } & 1) $7.6 \mathrm{e}^{0.34 t}( \pm 11 \%)$ \\
\hline & 2) $24 \mathrm{e}^{0.25 t}( \pm 2.9 \%)$ \\
\hline & 3) $22 t^{2}+4100 t-11 \times 10^{4}( \pm 1.2 \%)$ \\
\hline & 4) $2800 t-14,000( \pm 0.58 \%)$ \\
\hline & 5) $23,000 \sqrt{t}-25,000( \pm 0.2 \%)$ \\
\hline \multirow[t]{5}{*}{ Spain (February 26) } & 1) $15 \mathrm{e}^{0.33 t}( \pm 8.1 \%)$ \\
\hline & 2) $230 \mathrm{e}^{0.19 t}( \pm 3.6 \%)$ \\
\hline & 3) $27 t^{2}+5900 t-14 \times 10^{4}( \pm 0.34 \%)$ \\
\hline & 4) $4600 t-50,000( \pm 0.64 \%)$ \\
\hline & 5) $51,000 \sqrt{t}-17 \times 10^{4}( \pm 0.16 \%)$ \\
\hline \multirow[t]{5}{*}{ Italy (February 21) } & 1) $60 \mathrm{e}^{0.33 t}( \pm 3.2 \%)$ \\
\hline & 2) $0.51 t^{4}-30 t^{3}+770 t^{2}-8500 t+35,000 \quad( \pm 1.5 \%)$ \\
\hline & 3) $7.8 t^{3}-760 t^{2}+30,000 t-38 \times 10^{4} \quad( \pm 0.61 \%)$ \\
\hline & 4) $3800 t-45,000( \pm 0.61 \%)$ \\
\hline & 5) $39,000 \sqrt{t}-12 \times 10^{4}( \pm 0.3 \%)$ \\
\hline \multirow[t]{3}{*}{ Japan (February 15) } & 1) $58 \mathrm{e}^{0.093 t}( \pm 4.6 \%)$ \\
\hline & 2) $0.38 t^{3}-38 t^{2}+1400 t-16,000( \pm 1.9 \%)$ \\
\hline & 5) $330 t-10,000( \pm 1.2 \%)$ \\
\hline \multirow[t]{2}{*}{ India (March 04) } & 1) $17 \mathrm{e}^{0.16 t}( \pm 7.1 \%)$ \\
\hline & 2) $28 t^{2}-1300 t+17,000( \pm 0.86 \%)$ \\
\hline
\end{tabular}

functions are listed in Table 1, as well as exhibited in all the plots. We have computed the best-fit curves using Python's polyfit function, and the errors as the relative errors between the original data and the fitted data.

In the early phase, all the curves exhibit exponential growth as $I(t)=A \exp (\beta t)$, where $\beta$ is the growth rate. Interestingly, the $I(t)$ plots for USA, Spain, Germany, and France require two exponential functions for the fits. For example, Germany's data requires two functions, $\exp (0.34 t)$ and $\exp (0.25 t)$. Note that the growth rate $\beta$ varies for different countries, which is because $\beta$ depends on various factors such as immunity level of the population, climate, local policy decisions (lockdown, social distancing, testing intensity), etc.

Larger the $\beta$, larger the growth rate for the infection. Also, the inverse of the constant $\beta$ yields the growth time scale. In fact, in the exponential phase, the number of cases double in time $T=(\log 2) / \beta$. For South Korea, $\beta=0.67$, hence, $T \approx 1$; that is, $I(t)$ for South Korea doubled every day in the early phase (February 18 to February 23 ). The doubling time 
for India in the exponential phase was $\log (2) / 0.16 \approx 4.3$ days. Note that for the exponential regime, $\dot{I} \approx \beta I$.

After the exponential phase, the curves transition to power laws, polynomials to be more precise. The curve for China exhibits three power laws: $t^{2}, t$, and then $\sqrt{t}$, after which $I(t)$ saturates. Among the nine nations, China and South Korea have flattened their epidemic curves, while Italy, Spain, France, and Germany have reached $\sqrt{t}$ regime after transitioning through a series of power law regimes. These nations have almost flattened their epidemic curves. Note that some nations exhibit $t^{3}$ and $t^{4}$ power laws as well. We also remark that the best-fit curves are polynomials (see Table 1); the power laws mentioned above and those indicated in the plots are the leading-order terms of the respective polynomials. Among these countries, USA, Japan, and India are yet to rach $\sqrt{t}$ regime.

Figure 1 also contains plots for $\dot{I}$, derivatives of $I(t)$, that represents daily new count of infections. Similar to $I(t)$, $I$ increases exponentially in the beginning. After this, we observe a transition to power law regimes. For the power law $I(t) \sim B t^{n}$, we derive that $\dot{I} \sim I^{1-1 / n}$. Clearly, this slope is suppressed compared to the exponential regime by a factor of $I^{-1 / n}$. Note however that the epidemic growth in the power law regime is still very significant because $I(t)$ is large. For large $n$ (e.g., 4 or 5), $I \propto I$, which is same as the formula for the exponential growth (refer to the $t^{4}$ regime of Italy). Also note that in the linear regime, $\dot{I}$ is constant, implying a constant number of new cases every day. We can estimate the doubling time using the best-fit functions.

In Fig. 1, in the exponential regimes, $I(t)$ and $\dot{I}(t)$ curves run almost parallel to each other because $\dot{I} \approx \beta I$. In contrast, in the power law regimes, $\dot{I}(t)$ deviates from being parallel to $I(t)$, consistent with the suppression in $\dot{I}(t)$ mentioned in the previous paragraph. However, for large $n, \dot{I}$ exhibits a marginal deviation from the form $\dot{I} \approx \beta I(t)$.

We can combine the above ingredients into a comprehensive picture for the epidemic forecast, specially for flattening or saturating the $I(t)$ curve that is prime objective for most affected nations. As illustrated in the schematic diagram of Fig. 2, the $I(t)$ curve follows four stages: $\exp (\beta t), t^{n}$, $t$, and constant, which are represented by $\mathrm{S} 1, \mathrm{~S} 2, \mathrm{~S} 3$, and $\mathrm{S} 4$ respectively. It is an elementary observation that the $I(t)$ curve transitions from a convex form to a concave form. Such a simple observation of the data reveals insights into the temporal evolution of the epidemic. For example, before flattening of $I(t)$, we look for flattening of the growth rate $\dot{I}(t)$, which is the third stage in Fig. 2. Our preliminary studies show that the the above picture of evolution is also applicable to SARS and EBOLA epidemics (Ma 2020; World Health Organization 2003).

Recently, Prakash et al. (2020) studied the phase space portraits, that is, $I$ vs. I plots and observed the phase-space curves to be linear. This is natural for the exponential growth

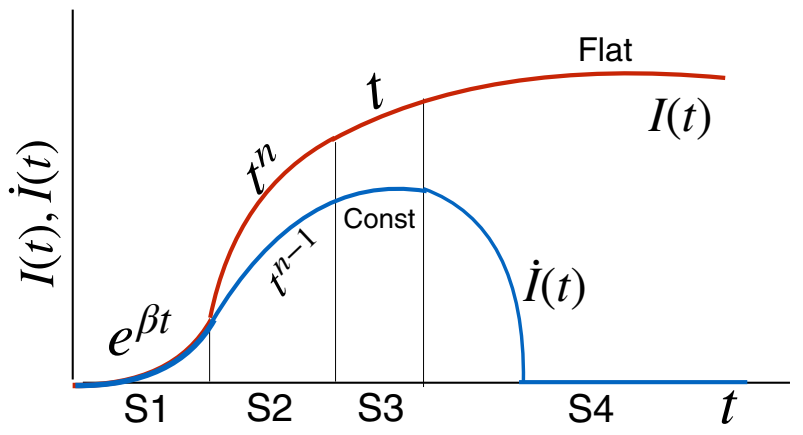

Fig. 2 (color online) For COVID-19 epidemic: Schematic plots for $I(t)$ and its derivative $\dot{I}(t)$ vs. $t$. S1, S2, S3, S4 represent the four stages of the epidemic: exponential growth in count $(\exp (\beta t))$, power law growth $\left(t^{n}\right)$, linear growth $(t)$, and flat

$(\dot{I} \propto \beta I)$, as well as for the power-law growth with large exponent $n$, because $\dot{I} \sim I^{1-1 / n}$.

Note that the above features of Fig. 2 appear in almost all epidemic models. Wu et al. (2020) considered a model with $\dot{I}=r I^{p}\left(1-(I / \kappa)^{\alpha}\right)$, where $r, p, \kappa, \alpha$ are free parameters, to provide a fit to the epidemic curve for China. In our paper, the focus is on the data itself, rather than models that may involve many parameters.

\section{Conclusions and Discussion}

The main result of our paper is the emergence of power laws between the exponential regime and flattening of the COVID-19 epidemic. We believe that the appearance of the power law is related to the lockdowns, imposed social distancing, and long distance travels by asymptomatic carriers. Considering strong similarities between the rumor spreading and epidemics (Daley and Gani 2001), the long-distance travels and power-law regimes may also play a major role in rumor spreading. Note that the social media and internet provide means for fast transmission of rumor.

The aforementioned power-law growth of epidemic appears to have similarities with turbulent diffusion or Taylor diffusion, which is faster than molecular diffusion (Taylor 1954; Leslie 1973; Sreenivasan 2018; Verma 2018, 2019). In turbulent diffusion, the separation between two particles, $D(t)$, increases as $t^{3 / 2}$, and $\dot{D} \sim D^{1 / 3}$. The relative velocity between the particles, $\dot{D}$, increases with time because larger eddies have larger speeds. This feature has a qualitative resemblance with aforementioned long-distance travels by asymptotic carriers.

There is possibly another connection of COVID-19 epidemic with turbulence and critical phenomena. In early stages, the epidemic spreads via contacts between infected and susceptible individuals. However, once the epidemic has 
spread widely, then indirect transmissions-contacts with infected surfaces, public transport, air-begin to play an important role in the epidemic growth. Such transmissions are referred to as community spread or transmission. This is analogous to interactions among clusters of molecules in phase transition, and those among large fluid vortices in turbulence. Such interactions are responsible for the dynamic scaling in phase transition, and for the aforementioned turbulence diffusion (Wilson and Kogut 1974; Täuber 2017; Taylor 1954; Leslie 1973; Sreenivasan 2018; Verma 2018, 2019). Super spreading of COVID-19 and the power-law regime of $I(t)$ may be connected to the above phenomena. Note however that the community spread could also contribute in the exponential growth phase; the two exponential regimes in Fig. 1 may be due to these reasons. These issues need further exploration.

The $I(t)$ plots of Fig. 1 exhibit different values for the growth rate $\beta$ and polynomials. These constants depend on various factors, such as immunity levels of the population, climate conditions, extent of lockdowns, levels of testing, social distancing, etc. The slowed progress of the epidemic in India may be due to the stricter and early lockdown. We also believe that a careful study of the $I(t)$ curves may help in forecasting when the epidemic curve will flatten.

Now, we summarize our findings. The COVID-19 realtime data of infected individuals, $I(t)$, contains useful information that may help forecast the development of the epidemic. We conjecture that the power law growth of $I(t)$ may be due to the epidemic transmission by asymptomatic carriers traveling long distances, and/or due to community spread.

Acknowledgements The authors thank Shashwat Bhattacharya help in early works. We also thank Shayak Bhattacharya, Prateek Sharma, and Anurag Gupta for useful discussions. Ali Asad is supported by Indo-French (CEFIPRA) project 6104-1, and Soumyadeep Chatterjee is supported by INSPIRE fellowship (IF180094) of Department of Science \& Technology, India.

\section{References}

Bjørnstad ON (2018) Epidemics: models and data using R. Springer, Berlin

Blasius B (2020) Power-law distribution in the number of confirmed COVID-19 cases. arXiv:2004.00940

Cherednik I, Hill UC (2020) Momentum managing epidemic spread and Bessel functions. arXiv:2004.06021

Chinazzi M, Davis JT, Ajelli M, Gioannini C, Litvinova M, Merler S, Piontti A Pastore y, Mu K, Rossi L, Sun K, Viboud C, Xiong X, Yu H, Halloran ME, Longini Jr IM, Vespignani A (2020) The effect of travel restrictions on the spread of the 2019 novel coronavirus (COVID-19) outbreak. Science (preprint)

Daley DJ, Gani J (2001) Epidemic modelling: an introduction. Cambridge University Press, Cambridge

Hellewell J, Abbott S, Gimma A, Bosse NI, Jarvis CI, Russell TW, Munday JD, Kucharski AJ, Edmunds J (2020) Feasibility of controlling COVID-19 outbreaks by isolation of cases and contacts. Lancet Glob Health. https://doi.org/10.1016/S2214

Johns Hopkins University (2020) Corona Resource Center. https:// coronavirus.jhu.edu/map.html

Kermack WO, McKendrick AG (1927) A contribution to the mathematical theory of epidemics. Proc R Soc A 115(772):700

Komarova NL, Wodarz D (2020) Patterns of the COVID19 epidemic spread around the world: exponential vs power laws. medrxiv. org. https://doi.org/10.1101/2020.03.30.20047274

Labadin J, Hong BH (2020) Transmission Dynamics of 2019-nCoV in Malaysia. medrxiv.org. https://doi. org/10.1101/2020.02.07.20021188

Leslie DC (1973) Developments in the theory of turbulence. Clarendon Press, Oxford

Li R, Pei S, Chen B, Song Y, Zhang T, Yang W, Shaman J (2020) Substantial undocumented infection facilitates the rapid dissemination of novel coronavirus (SARS-CoV2). Science. https ://doi.org/10.1126/science.abb3221

Ma J (2020) Estimating epidemic exponential growth rate and basic reproduction number. Infect Dis Model 5:129

Manchein C, Brugnago EL, da Silva RM, Mendes CFO, Beims MW (2020) Strong correlations between power-law growth of COVID-19 in four continents and the inefficiency of soft quarantine strategies. arXiv:2004.00044

Mandal S, Bhatnagar T, Arinaminpathy N, Agarwal A, Chowdhury A, Murhekar M, Gangakhedkar R, Sarkar S (2020) Prudent public health intervention strategies to control the coronavirus disease 2019 transmission in India: a mathematical model-based approach. Indian J Med Res 151(2):190

Min Y, Jin X, Ge Y, Chang J (2013) The role of community mixing styles in shaping epidemic behaviors in weighted networks. PLoS One. https://doi.org/10.1371/journal.pone.0057100

Peng L, Yang W, Zhang D, Zhuge C, Hong L (2020) Epidemic analysis of COVID-19 in China by dynamical modeling. medarxiv. org. https://doi.org/10.1101/2020.02.16.20023465

Prakash MK, Kaushal S, Bhattacharya S, Chandran A, Kumar A, Ansumali S (2020) A minimal and adaptive prediction strategy for critical resource planning in a pandemic. medrxiv.org. https ://doi.org/10.1101/2020.04.08.20057414

Schüttler J, Schlickeiser R, Schlickeiser F, Kröger M (2020) Covid19 predictions using a Gauss model, based on data from April 2. preprints.org

Shayak B, Sharma MM, Rand RH, Singh AK, Misra A (2020) Transmission dynamics of COVID-19 and impact on public health policy. medrxiv.org. https://doi.org/10.1101/2020.03.29.20047 035

Shim E, Tariq A, Choi W, Lee Y, Chowell G (2020) Transmission potential and severity of COVID-19 in South Korea. Int J Infect Dis (preprint)

Sreenivasan KR (2018) Turbulent mixing: a perspective. PNAS $371: 201800463$

Täuber UC (2017) Phase transitions and scaling in systems far from equilibrium. Annu Rev Condens Matter Phys 8(1):185

Taylor GI (1954) The dispersion of matter in turbulent flow through a pipe. Proc R Soc A 223(1):446

Verma MK (2018) Physics of buoyant flows: from instabilities to turbulence. World Scientific, Singapore

Verma MK (2019) Energy transfers in fluid flows: multiscale and spectral perspectives. Cambridge University Press, Cambridge

Wilson KG, Kogut J (1974) The renormalization group and the $\varepsilon$ expansion. Phys Rep 12(2):75

World Health Organization (2003) Consensus document on the epidemiology of severe acute respiratory syndrome (SARS). Tech. rep

WorldOMeter (2020) https://www.worldometers.info/coronavirus/ 
Wu K, Darcet D, Wang Q, Sornette D (2020) Generalized logistic growth modeling of the COVID-19 outbreak in 29 provinces in China and in the rest of the world. arXiv.org

Ziff AL, Ziff RM (2020) Fractal kinetics of COVID-19 pandemic. medrxiv.org. https://doi.org/10.1101/2020.02.16.20023820
Publisher's Note Springer Nature remains neutral with regard to jurisdictional claims in published maps and institutional affiliations. 